Gut, 1973, 14, 689-696

\title{
The electrical and motor actions of gastrointestinal hormones on the duodenum in man
}

\author{
W. E. WATERFALL, H. L. DUThiE, AND B. H. BROWN \\ From the Departments of Surgery and Medical Physics, University of Sheffield, Sheffield
}

SUMMARY The electrical and motor activity was recorded from the duodenum in 16 conscious human subjects in resting conditions. The main electrical wave form variously known as the pacesetter potential or slow wave was constantly present and periodically accompanied by a second electrical trace consisting of rapid bursts of fast waves or action potentials which were related to motor waves. Secretin and the synthetic analogue of gastrin, pentagastrin, were given in separate tests and their complementary actions on myoelectrical activity are reported. In general, secretin (in six subjects) depressed and pentagastrin (in eight subjects) enhanced motor activity and bursts of action potentials.

Over the past four decades myoelectrical activity has been recorded from the muscular layer of the human alimentary tract (Alvarez, 1929; Daniel, Wachter, Honour, and Bogoch, 1958; Christensen, Schedl, and Clifton, 1966; Couturier, Roze, Paolaggi, and Debray, 1971 ; Kwong, Brown, Whittaker, and Duthie, 1971a). Two types of electrical activity are recognized: an ever-present cyclical change in potential called the slow wave or pacesetter potential and intermittent activity consisting of action potential bursts superimposed on the slow wave. Action potential bursts are temporarily related to motor activity, each burst accompanying a motor wave.

The relationship between electrical activity and motility is clearly established but to date there has been little information concerning the overall regulation of myoelectrical activity. However, Kwong, Brown, Whittaker, and Duthie (1971b) demonstrated the potentiation of the pacesetter potential (PSP) and motor waves in the normal human stomach by pentagastrin and the inhibitory effects of secretin. More recently the effects of these two hormones in the terminal ileum in patients with an ileostomy have been reported (Waterfall, Brown, Duthie, and Whittaker, 1972). We now report the modifying influence of pentagastrin and secretin on the electrical and motor activity of the duodenum in man.

\section{Method}

A heterogeneous group of individuals comprising Received for publication 3 May 1973. medical students, patients with biliary disease, and others awaiting elective surgery for hernia and haemorrhoids, gave informed consent to be subjects of the study. Electrical and motor waves were recorded from the duodenum in 16 subjects, using a probe as described previously (Waterfall et al, 1972). The probe consisted of a modified polythene tube armed with a stainless steel electrode $0.25 \mathrm{~mm}$ diameter projecting $1 \mathrm{~mm}$ through a side hole (fig 1).

\section{ELECTRICAL ACTIVITY}

A negative pressure of $20 \mathrm{~cm} \mathrm{Hg}$ was applied to the proximal end of the probe to attach the electrode to the bowel wall. The electrode was surrounded by a rubber cup which diminished movement artefact when the bowel contracted vigorously. Unipolar records were made using an indifferent surface

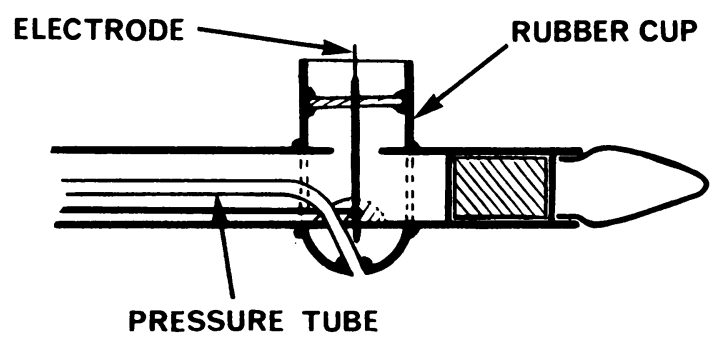

Fig 1 The tip of the probe illustrating the electrode and the opening of the open tip catheter diametrically opposed. 
electrode placed on the scarified abdominal skin in a position chosen by trial and error to diminish ECG artefact. An AC amplifier with a frequency response of $0.02 \mathrm{~Hz}$ to $1 \mathrm{kHz} \pm 3 \mathrm{~dB}$ was used to amplify the electrical record which was then displayed on ultraviolet paper at a sensitivity of $3 \mathrm{~cm} /$ millivolt. Parallel outputs were led to the recorder, one passing via an electronic band pass filter, frequency range 9-14 cycles/min. Electrical and motor activity were also recorded on magnetic tape.

The frequency and amplitude of the main electrical waveform, the PSP, was measured and the presence of action potential bursts counted. The latter consisted of more rapid electrical oscillations superimposed on the pacesetter potential and their relationship to motor waves was noted.

\section{MOTOR ACTIVITY}

An open tip catheter system was used to detect changes in intraluminal pressure, the opening of the fine polythene tube being brought out opposite the electrode. The catheter was perfused with 0.15 molar saline at a rate of $10 \mathrm{ml} /$ hour. The proximal end of the catheter was connected via a three-way tap to a pressure transducer and intraluminal pressure changes were detected and displayed on the recorder. The duration of each pressure wave was measured and their sum expressed as a percentage of the total recording time (percentage motility).

A double-ended probe equipped with two electrodes and two pressure tips was used so that motor and electrical activity from two sites in the duodenum, $4 \mathrm{~cm}$ apart, were displayed in addition to the pneumogram.

\section{PROCEDURE}

Subjects were studied in a comfortable semirecumbent position on a couch after an overnight fast. The probe was stiffened with a Seldinger wire, and, after induction of pharyngeal anaesthesia with anaesthetic spray, was advanced into the stomach. Adjustment of the tube was achieved under fluoroscopic control so that the probe was positioned just proximal to the pylorus. The Seldinger wire was withdrawn and further advancement of the probe procured by spontaneous gastric and duodenal motor waves. If the tube had not passed into the duodenum after two hours the test was abandoned. A second tube was passed into the most dependent part of the stomach and aspirated continuously throughout each test. Sixteen patients were investigated in a total of 21 tests under resting conditions.

Pentagastrin in doses of 1.0 and $6 \mu \mathrm{g} / \mathrm{kg} \mathrm{hr}$ was given by continuous intravenous infusion to eight patients. Secretin was given by continuous intravenous infusion in separate tests in 0.25 and $1 \mathrm{unit} / \mathrm{kg}$ $\mathrm{hr}$ doses to six patients. These doses were selected because they produced the maximal effect on myoelectrical activity in the normal human stomach (Kwong et al, 1971b) and in the terminal ileum (Waterfall et al, 1972). Hormones were infused at each dose for a period of $\mathbf{4 0}$ minutes.

At the commencement of each test a saline infusion was instituted and recordings were made for 45 minutes. In those subjects who received hormones the infusion of either secretin or pentagastrin was substituted for the saline at this time. Two subjects received saline only for the total period of the test and the extended control period was observed.

The total recording time in the 15 patients was 22 hours during saline infusion. During secretin infusion the period was eight hours and during pentagastrin infusion was 11 hours.

In six patients undergoing cholecystectomy for gallstones four stainless steel electrodes were implanted subserosally on the first, second, and third parts of the duodenum and brought out through the right hypochondrial drain. Electrical records only were made during saline and hormone infusion from the third to the seventh post-operative days. The electrodes were then removed with the drain.

On magnetic tape data were processed using Biomac 1000 and ICL correlator, auto and cross correlation functions were calculated. Standard tests of statistical significance were used.

\section{Results}

\section{RESTING STUDIES}

In the fasting state during saline infusion in 16 patients, the frequency of the pacesetter potential varied between 11.2 and 12.6 cycles/minute (mean $11 \cdot 8 \pm 0 \cdot 12$ ). The frequency of the PSP varied ( \pm 0.4 cycles/minute) from minute to minute but the average frequency was constant for each test and also in separate tests in any individual. The amplitude of the pacesetter potential waxed and waned with a range of 0.2 to 1.6 millivolts (fig 2). Action potential bursts were seen superimposed on the pacesetter potential and were coexistent with pressure waves (fig 3-detail to show action potential bursts). Additionally the 3 cycle/minute PSP characteristic of the stomach was detected in the proximal duodenum and readily demonstrable using a second electronic band filter (2-5 cycles/minute, fig 4).

Motor waves were recorded in all subjects. The percentage activity in the fasted state ranged from 1 to $45 \%$ (mean $=16 \%$ ). Individuals who were tested more than once showed $<10 \%$ change in spontaneous motility. The two saline infusion control studies showed no significant shift in the average frequency of the PSP (maximum change 0.4 cycles) 


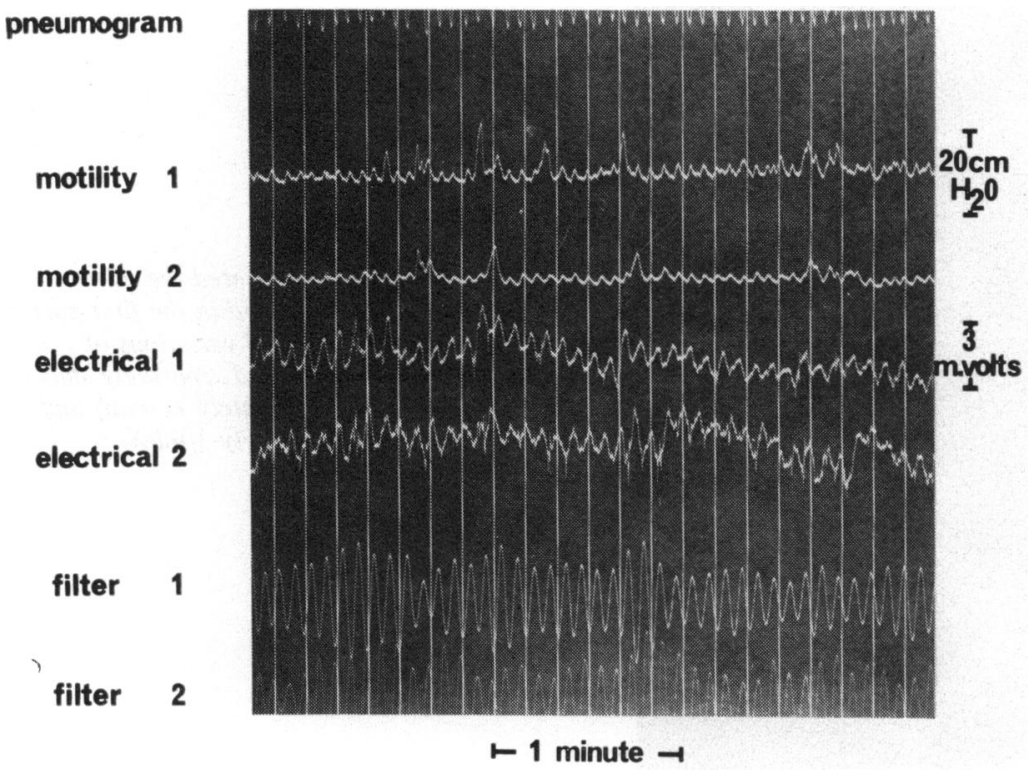

Fig 2 A record illustrating the cyclical nature of the pacesetter potential and action potential bursts during resting conditions. The pneumogram is proof that the pressure changes are not caused by respiration. The filtered electrical wave gives additional confirmation of the cyclical form of the pacesetter potential.

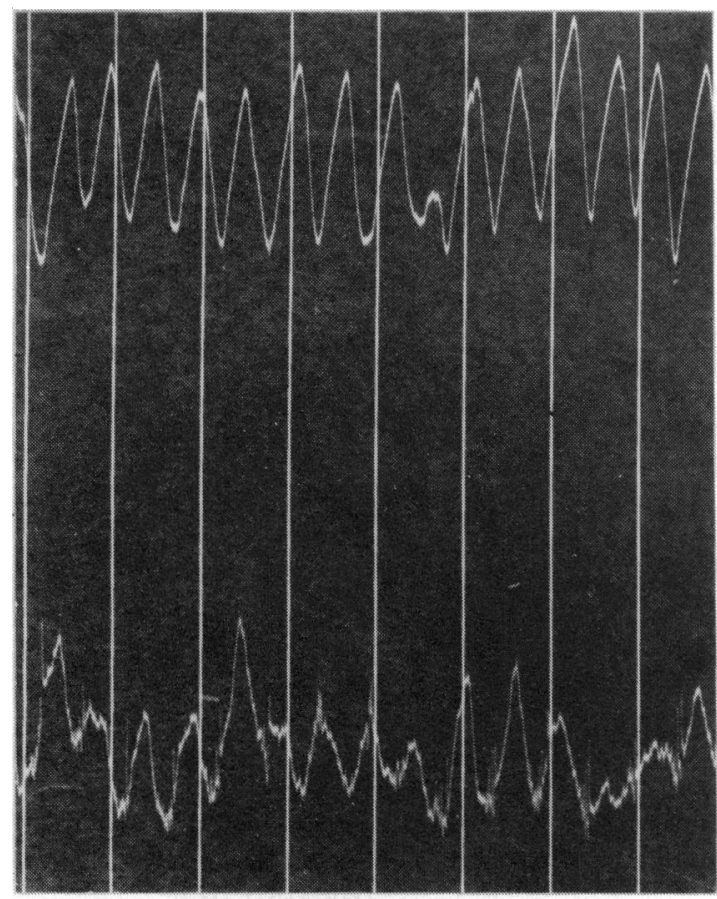

\section{pressure}

Fig 3 A detail of a record enlarged to show the relationship of action potential bursts on the electrical trace corresponding to the rise in pressure on the pressure trace.

\section{electrical}




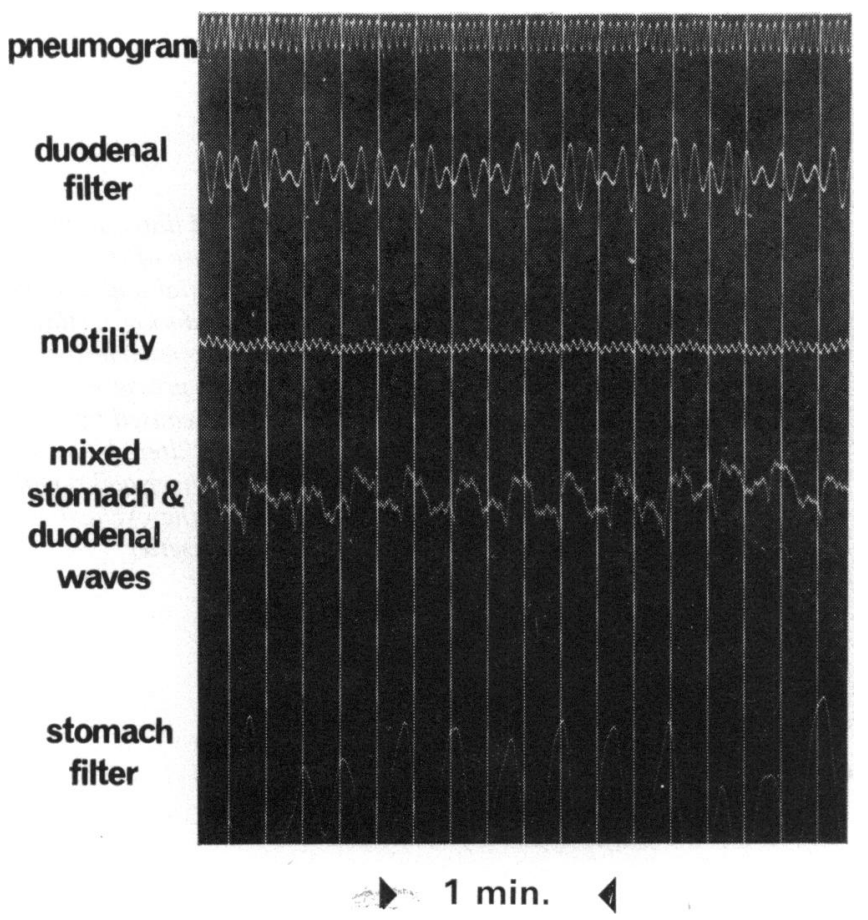

Fig 4 This record illustrated the mixed electrical patterns obtained in the first part of the duodenum. Filtered variations of these waves are shown and separately show the duodenum (approximately 12/min) and the stomach (approximately 3/min) pacesetter potentials.

minute) during successive 15 -minute periods over three hours. The change in percentage motility was less than $6 \%$ over consecutive 15 -minute periods.

The results presented are the changes produced by hormones in higher dosage. When the lower hormone dosage was given more than $90 \%$ of subjects produced a response which was qualitatively similar but numerically usually less than the higher dose response. The remaining subjects failed to respond to hormone infusion at the lower dose.

In the six patients with implanted electrodes in 14 tests under resting conditions, the pacesetter potential only was recorded. It was of the same order of magnitude as records obtained with the luminal probe and the mean frequency of the PSP was not significantly different. The frequency of the PSP was constant at all electrode sites in each individual patient. Maximum variation was 0.8 cycle and the mean frequency 11.6 cycles/minute \pm 0.15 . A frequency plateau existed in the first, second, and third parts of the duodenum. There was no constant phase relation between electrodes, but, using the cross-correlation technique, a time-dependent phase shift was observed. These findings differ from those in the dog duodenum where there is a frequency plateau with a constant phase shift (Duthie, Brown, Robertson-Dunn, Kwong, Whittaker, and Waterfall, 1972).

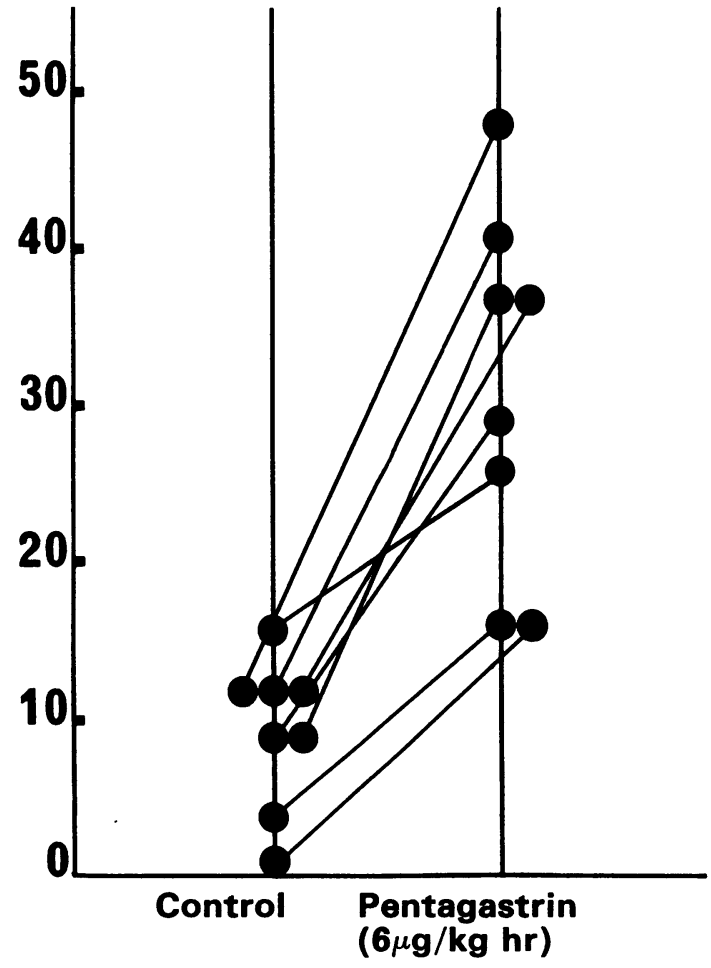

Fig 5 The increase in percentage motor activity produced by pentagastrin administration. 


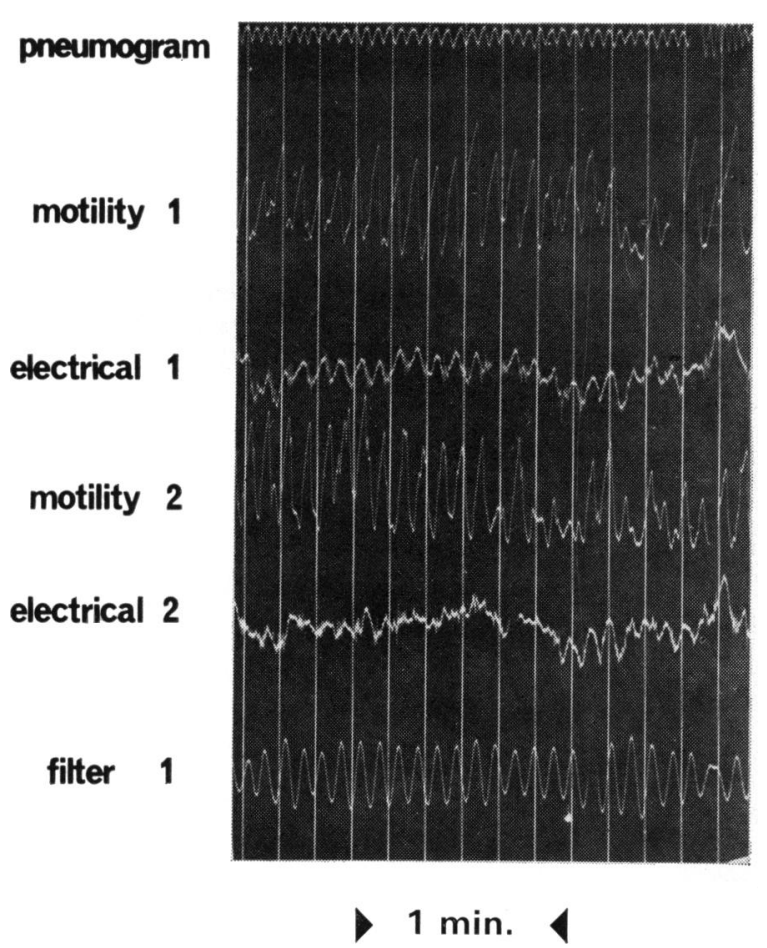

Fig 6 A record showing the increase in frequency of action potential bursts and increase in the number of pressure waves produced by pentagastrin administration.

\section{INFUSION OF HORMONES}

Secretin in a dose of $1 \mathrm{unit} / \mathrm{kg} \mathrm{hr}$ and pentagastrin in a dose of $6 \mu \mathrm{g} / \mathrm{kg} \mathrm{hr}$ produced a significant effect $(P<0.01)$ on the frequency of action potential bursts. Secretin reduced, and pentagastrin increased, the bursts of action potentials. These changes in action potential frequency were in direct proportion to changes in motility during infusion with each hormone. Pentagastrin increased the percentage motility in all eight subjects tested. This occurred simultaneously with the increase in frequency of action potential bursts (figs 5 and 6). In the six

\begin{tabular}{lll}
\hline Patient & Saline & Pentagastrin \\
\hline E & 12.2 & 12.2 \\
F & 11.3 & 11.5 \\
G & 12.0 & 12.1 \\
H & 11.4 & 11.3 \\
I & 12.1 & 12.0 \\
J & 11.0 & 11.1 \\
L & 11.3 & 11.4 \\
\hline
\end{tabular}

Table I Pacesetter potential frequency (cycles/min)

\begin{tabular}{lll}
\hline Patient & Saline & Secretin \\
\hline A & 12.2 & 12.3 \\
B & 11.9 & 11.8 \\
D & 11.6 & 11.7 \\
E & 11.6 & 11.4 \\
F & 11.2 & 11.3 \\
\hline
\end{tabular}

Table II Pacesetter potential frequency (cycles/min)

subjects who received secretin all showed a significant reduction $(P<0.05)$ in the frequency of motor waves (figs 7 and 8). Again this change occurred concomitantly with a reduction in the frequency of action potential bursts. However, the frequency of the PSP did not change significantly during infusion with either hormone (tables I and II).

Four of the patients with implanted electrodes were given the same doses of secretin (four subjects) and pentagastrin (four subjects) on separate days. When hormone infusion was substituted for saline infusion there was no change in PSP frequency.

Continuous aspiration of the gastric contents dur-

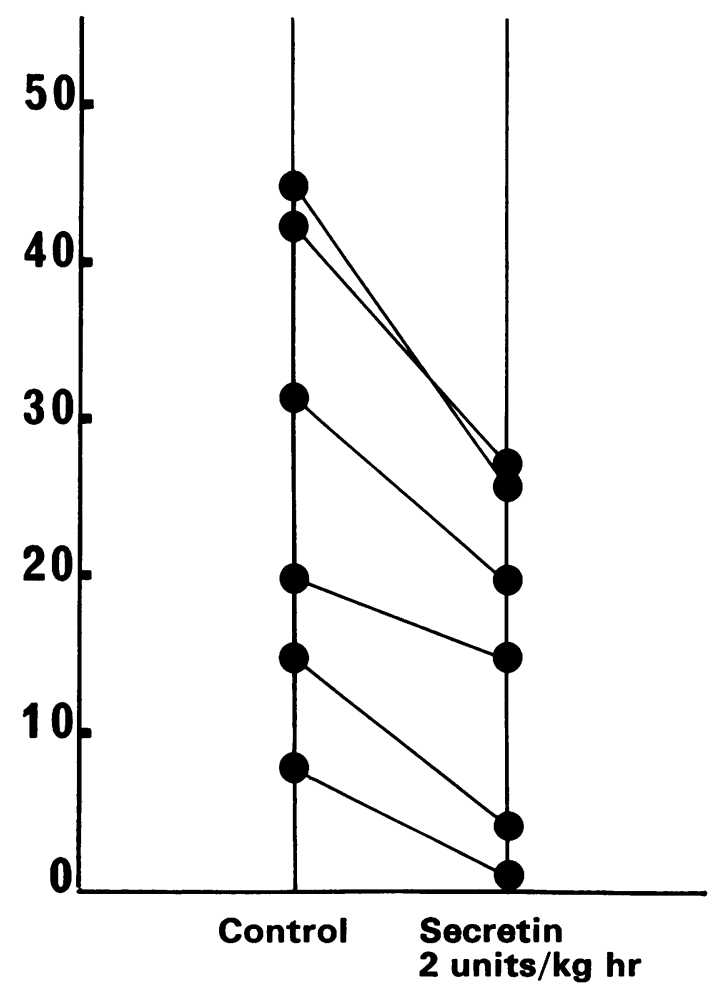

Fig 7 The decrease in percentage motor activity produced by secretin infusion. 


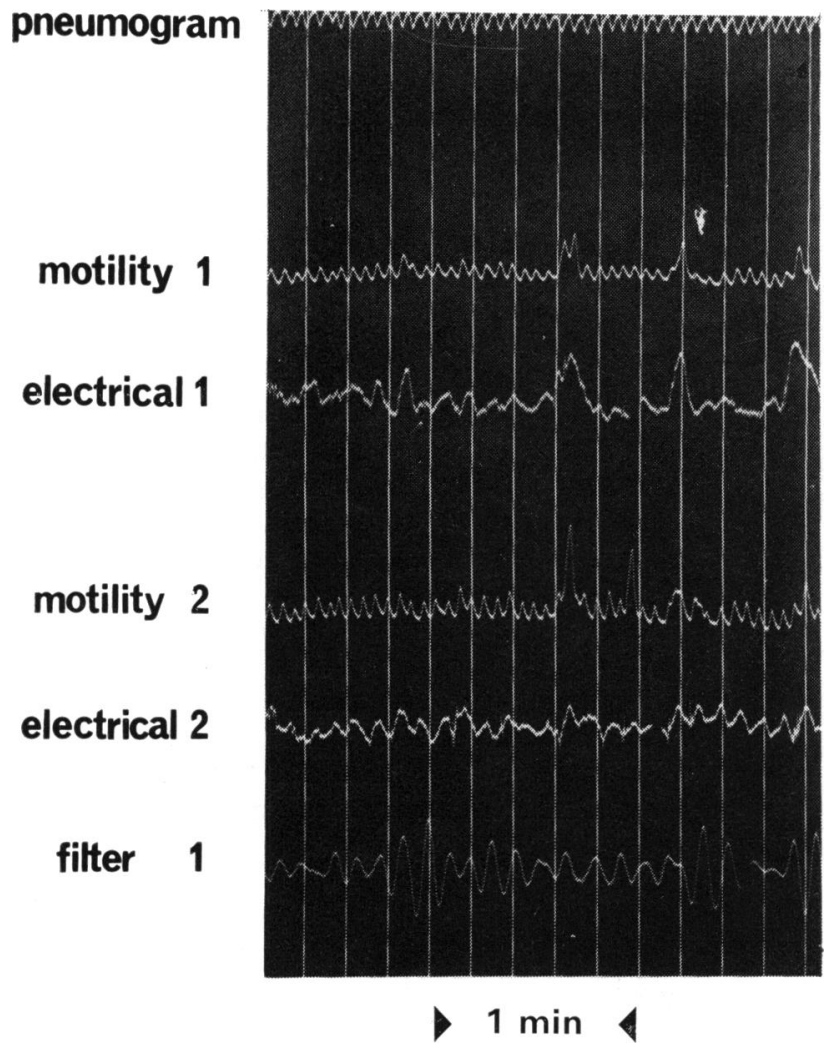

Fig 8 A record showing the decrease in frequency of action potential bursts and the decrease in pressure wave activity produced by secretin infusion.

ing each test prevented material entering the duodenum from the stomach and avoided stimulation of duodenal mucosal receptors. Thus the duodenal motility detected was as near as possible basal activity or activity due to blood-borne humoral agents.

\section{Discussion}

The duodenal frequency of the pacesetter potential, its amplitude, and the basal existence of action potential bursts related temporarily to motor waves under resting conditions confirms the findings of others (Christensen et al, 1966; Monges and Salducci, 1970). The mean percentage motor activity of $16 \%$ agrees well with the findings of Fink (1959), who demonstrated a mean percentage motor activity of $15 \%$ in the resting human duodenum using the open tip catheter technique. The frequency and the amplitude of the PSP was not significantly different when recorded from either the mucosal or serosal electrodes showing that the luminal probe is a reliable recording instrument. Action potential bursts were rarely recorded from the serosal electrode but readily seen during the probe studies. It is probable that the depth of the electrode in the bowel wall is of some importance in this matter for Daniel et al (1958) have shown that the maximum amplitude of action potentials is recorded in or close to the enteric plexuses.

The waxing and waning of the amplitude of the PSP was noted in all patients and in six subjects the relationship of action potential bursts to amplitude was investigated. In each record the pacesetter potential waves were measured and the frequency of action potential bursts was noted. A mean value of percentage frequency of action potential bursts was plotted against amplitude of the PSP and is illustrated in figure 9. From these measurements it would appear that a level of negativity of the PSP greater than $\mathbf{0 . 2}$ millivolts is required to produce action potentials. Intracellular recordings (Bulbring, 1973, personal communication) have not shown any notable amplitude change comparable with our extracellular studies. It is conceivable that contraction of the bowel causes shift of the electrode allowing it to be influenced by more smooth muscle cells. The resultant of the electrode 'seeing' more smooth muscle cells might effectively be an increase in the amplitude of the pacesetter potential. Though 


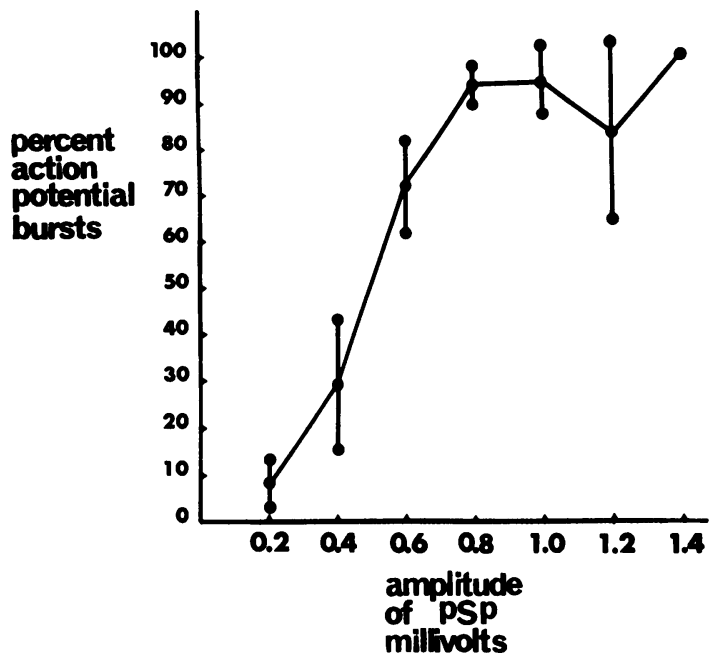

Fig 9 A graph showing the percentage frequency of action potential bursts plotted against pacesetter potential amplitude. It illustrates increase in percentage of action potential bursts with increasing amplitude of pacesetter potential.

this explanation seems feasible it does not explain our findings of amplitude change when the bowel is inactive.

To date a variety of agents have been shown to effect small bowel myoelectrical activity both in vivo and in vitro (McMahon, Code, Sauer, and Bargen, 1949; Daniel, Carlow, Wachter, Sutherland, and Bogoch, 1959; Bennett and Whitney, 1966; Neely, 1968). The effects of caerulein and serotonin in stimulating small bowel motility and also causing a decrease in PSP frequency have been demonstrated by Labo, Barbara, Lanfranchi, Bortolotti, and Miglioli (1971). The study presented here shows that secretin and pentagastrin have, in addition to their secretory properties, influence on the motor and electrical activity of the duodenum. However, the effects in vivo of these substances must remain speculative. The augmentation of motor waves and of the bursts of action potentials on the electrical recordings from the duodenum during the intravenous infusion of pentagastrin are similar to our findings in the ileum (Waterfall et al, 1972) and the gastric antrum. Although an increase in the frequency of the PSP waves was also seen in the stomach (Kwong et al, 1971a; Monges et al, 1971) this was not observed in the duodenum or terminal ileum.

The stimulatory effect of gastric acid in the duodenum causing release of duodenal hormones such as cholecystokinin is well known. Since aspiration of gastric juice from the stomach is rarely complete, the myoelectrical changes recorded could have been due to endogenous release of duodenal hormones by gastric acid. However, the increase in gastric acid secretion following pentagastrin infusion usually begins 10 minutes after the administration of the hormone. The duodenal motor and electrical changes were rapid and occurred within two minutes of commencement of hormone infusion. It seems plausible therefore that the changes observed were due to infused pentagastrin and not to endogenously released hormones since the changes reported occurred rapidly and certainly sooner than the secretion of gastric juice from the stomach.

The stimulatory effects of gastric juice on duodenal motility were minimized by aspiration of the stomach throughout the tests but pancreatic juice was not aspirated from the duodenum. However, the patterns of response to secretin infusion are similar in the stomach and terminal ileum and the flow of pancreatic juice does not appear to have introduced any artefacts.

It is interesting to speculate whether the hormones studied have a physiological role to play in the control of duodenal motility. It would seem unlikely as they show only a $50 \%$ change in activity when pharmacological doses of hormones are given. But in maximal stimulatory periods after a meal they may be expected to promote mixing in the duodenum and also to allow more time for the action of pancreatic juice on the contents of the duodenum. Obviously both hormones may be circulating at the same time and their joint action would be of interest.

Technical assistance was supported in part by the Medical Research Council.

\section{References}

Alvarez, W. C. (1929). Physiological studies on the motor activities of the stomach and bowel in man. Amer. J. Physiol., 88, 650-662.

Bennett, A., and Whitney, B. (1966). A pharmacological study of the motility of the human gastrointestinal tract. Gut, 7, 307-316.

Christensen, J., Schedl, H. P., and Clifton, J. A. (1966). The small intestinal basic electrical rhythm (slow wave) frequency gradient in normal men and in patients with a variety of diseases. Gastroenterology, 50, 309-315.

Couturier, D., Roze, C., Paolaggi, J., and Debray, C. (1971). Electrical activity of the normal human stomach: a comparative study of recordings obtained from the serosal and mucosal sides. $R$. $C$. rom. Gastro-ent., 3, 130.

Daniel, E. E., Carlow, D. R., Wachter, B. T., Sutherland, W. H., and Bogoch, A. (1959). Electrical activity of the small intestine. Gastroenterology, 37, 268-281.

Daniel, E. E., Wachter, B. T., Honour, A. J., and Bogoch, A. (1958). The relationship between electrical and mechanical activity of the small intestine in dog and man. Canad. J. Biochem., 38, 777-801.

Duthie, H. L., Brown, B. H., Robertson-Dunn, B., Kwong, N. K., Whittaker, G. E., and Waterfall, W. E. (1972). Electrical activity in the gastroduodenal area-slow waves in the proximal duodenum. A comparison of man and dog. Amer. J. dig. Dis., 17, 344-351.

Fink, S. (1959). The intraluminal pressures in the intact human intestine. Gastroenterology, 36, 661-671. 
Kwong, N. K., Brown, B. H., Whittaker, G. E., and Duthie, H. L. (1971a). Electrical activity of the gastric antrum in man. Brit. J. Surg., 57, 913-916.

Kwong, N. K., Brown, B. H., Whittaker, G. E., and Duthie, H. L. (1971b). Response of the electrical activity, motor activity and acid secretion of the human stomach. to pentagastrin and histamine stimulation Scand. J. Gastroent., 6, 145-153.

Labo, G., Barbara, L., Lanfranchi, G. A., Bortolotti, M., and Miglioli, M. (1971). Modifications of the electrical activity of human intestine after 5-HT ane caerulein. R. C. rom. Gastro-ent., 3, 130-131.

McMahon, J. M., Code, C. F., Sauer, W. G., and Bargen, J. A. (1949).
A study of the action of prostigmine of the bowel of human beings. Gastroenterology, 12, 970-977.

Monges, H., and Salducci, J. (1970). Etude électrolyographique de motricité duodénale chez l'homme normal. Arch. Mal. Appar. dig., 59, 19-28.

Neely, J. (1968). Comparison of the effects of a gastrin extract and a synthetic pentapeptide in gastrointestinal motility in the cat. Gut, 8, 242-248.

Waterfall, W. E., Brown, B. H., Duthie, H. L., and Whittaker, G. E. (1972). The effects of humoral agents on the myoelectrical activity of the terminal ileum. Gut, 13, 528-534. 